International Electronic Journal of Algebra

Volume 29 (2021) 211-222

DOI: $10.24330 /$ ieja. 852234

\title{
A GENERALIZATION OF THE ESSENTIAL GRAPH FOR MODULES OVER COMMUTATIVE RINGS
}

\author{
F. Soheilnia, Sh. Payrovi and A. Behtoei \\ Received: 18 April 2020; Revised: 27 July 2020; Accepted: 14 August 2020 \\ Communicated by Burcu Üngör \\ Abstract. Let $R$ be a commutative ring with nonzero identity and let $M$ be \\ a unitary $R$-module. The essential graph of $M$, denoted by $E G(M)$ is a simple \\ undirected graph whose vertex set is $Z(M) \backslash \operatorname{Ann}_{R}(M)$ and two distinct vertices \\ $x$ and $y$ are adjacent if and only if $\operatorname{Ann}_{M}(x y)$ is an essential submodule of $M$. \\ Let $r\left(\operatorname{Ann}_{R}(M)\right) \neq \operatorname{Ann}_{R}(M)$. It is shown that $E G(M)$ is a connected graph \\ with $\operatorname{diam}(E G(M)) \leq 2$. Whenever $M$ is Noetherian, it is shown that $E G(M)$ \\ is a complete graph if and only if either $Z(M)=r\left(\operatorname{Ann}_{R}(M)\right)$ or $E G(M)=K_{2}$ \\ and $\operatorname{diam}(E G(M))=2$ if and only if there are $x, y \in Z(M) \backslash \operatorname{Ann}_{R}(M)$ and $\mathfrak{p} \in$ \\ $\operatorname{Ass}_{R}(M)$ such that $x y \notin \mathfrak{p}$. Moreover, it is proved that $\operatorname{gr}(E G(M)) \in\{3, \infty\}$. \\ Furthermore, for a Noetherian module $M$ with $r\left(\operatorname{Ann}_{R}(M)\right)=\operatorname{Ann}_{R}(M)$ it \\ is proved that $\left|\operatorname{Ass}_{R}(M)\right|=2$ if and only if $E G(M)$ is a complete bipartite \\ graph that is not a star.
}

Mathematics Subject Classification (2020): 05C25, 13C99

Keywords: Prime submodule, essential submodule, essential graph

\section{Introduction}

The concept of the zero-divisor graph of a commutative ring was introduced and studied by I. Beck in [6]. Subsequently, D. F. Anderson and P. S. Livingston in [2] studied and investigated the concept of zero-divisor graph on nonzero zerodivisors of a commutative ring. Let $R$ be a commutative ring and let $Z(R)$ be its set of zero-divisors. The zero-divisor graph of $R$, which is the graph with vertex set $Z^{*}(R)=Z(R) \backslash\{0\}$ and two distinct vertices $x$ and $y$ are adjacent if and only if $x y=0$, has been studied by many authors (see $[1,3,4]$ ). Variations of the zerodivisor graph are created by changing the vertex set, the edge condition, or both. The essential graph of $R$ is a variation of the zero-divisor graph that changes the edge condition, and is introduced and studied in [10]. The essential graph of $R$ is a simple undirected graph, denoted by $E G(R)$, with vertex set $Z^{*}(R)$ and two distinct vertices $x$ and $y$ are adjacent if and only if $\operatorname{Ann}_{R}(x y)$ is an essential ideal 
of $R$. Recently, a lot of research (e.g., $[5,7,8,11,12])$ has been devoted to the zerodivisor graph of a module (Definition 4.1). Let $M$ be an $R$-module and let $Z(M)$ be its set of zero-divisors. In this paper, we associate a graph to the module $M$, denoted by $E G(M)$, with vertex set $Z(M) \backslash \operatorname{Ann}_{R}(M)$ and two distinct vertices $x, y \in Z(M) \backslash \operatorname{Ann}_{R}(M)$ are adjacent if and only if $\operatorname{Ann}_{M}(x y)$ is an essential submodule of $M$. Before we state some results, let us introduce some graphical notations.

Let $G=(V(G), E(G))$ be a simple undirected graph, $V(G)$ and $E(G)$ are called vertex set and edge set of $G$, respectively. Let $x, y \in V(G)$. Whenever $x$ and $y$ are joint by an edge, it is denoted by $x-y$. The vertex $x$ is said to be a universal vertex if it is adjacent to every other vertex of $G$. The graph $G$ is connected if there is a path between any two distinct vertices. For vertices $x$ and $y$ of $G$, we define $\mathrm{d}(x, y)$ to be the length of a shortest path between $x$ and $y$ (if there is no path, then $\mathrm{d}(x, y)=\infty)$. The open neighborhood of a vertex $x$ is defined to be the set $N(x)=\{y \in V(G): \mathrm{d}(x, y)=1\}$. The diameter of $G$ is $\operatorname{diam}(G)=$ $\sup \{\mathrm{d}(x, y) \mid x, y \in V(G)\}$. A graph $G$ is complete if any two distinct vertices are adjacent and a complete graph with $n$ vertices is denoted by $K_{n}$. A bipartite graph is one whose vertex set can be partitioned into two subsets so that an edge has both ends in no subset. A complete bipartite graph is a bipartite graph in which each vertex is adjacent to every vertex that is not in the same subset. The complete bipartite graph with part sizes $m$ and $n$ is denoted by $K_{m, n}$. If $m=1$, then the bipartite graph is called star graph. The girth of $G$, denoted by $\operatorname{gr}(G)$ is the length of a shortest cycle contained in the graph (if there is no cycle, then $\operatorname{gr}(G)=\infty$ ).

Throughout this paper, $R$ is a commutative ring with nonzero identity and $M$ is a unitary $R$-module. Recall that $Z(M)=\{r \in R: r m=0$ for some $0 \neq m \in M\}$, $\operatorname{Ass}_{R}(M)=\left\{\mathfrak{p} \in \operatorname{Spec}(R): \mathfrak{p}=\operatorname{Ann}_{R}(m)\right.$ for some $\left.0 \neq m \in M\right\}, \operatorname{Ann}_{R}(M)=$ $\{r \in R: r M=0\}$ and $r\left(\operatorname{Ann}_{R}(M)\right)=\left\{x \in R: x^{t} \in \operatorname{Ann}_{R}(M)\right.$ for some $t \in$ $\mathbb{N}\}$. For $x \in R, \operatorname{Ann}_{M}(x)=\{m \in M: x m=0\}$. Let $\operatorname{Spec}_{R}(M)$ denote the set of prime submodules of $M$. Then $m$-Ass $\operatorname{As}_{R}(M)=\left\{P \in \operatorname{Spec}_{R}(M): P=\right.$ $\operatorname{Ann}_{M}(x)$ for some $\left.0 \neq x \in R\right\}$. For notations and terminologies not given in this paper, the reader is referred to [13].

Here is a brief summary of the paper. In the second section, for a Noetherian $R$-module $M$ with $r\left(\operatorname{Ann}_{R}(M)\right) \neq \operatorname{Ann}_{R}(M)$, we show that $E G(M)$ is a connected graph with $\operatorname{diam}(E G(M)) \leq 2$ and $\operatorname{gr}(E G(M)) \in\{3, \infty\}$ (Theorem 2.6). We show that $E G(M)$ is a complete graph if and only if either $Z(M)=r\left(\operatorname{Ann}_{R}(M)\right)$ or $E G(M)=K_{2}$ (Theorem 2.10). Whenever $r\left(\operatorname{Ann}_{R}(M)\right)=\operatorname{Ann}_{R}(M)$, among other 
things, we prove that $\left|\operatorname{Ass}_{R}(M)\right|=2$ if and only if $E G(M)$ is a complete bipartite graph that is not a star (Theorem 3.7). In the fourth section, for a Noetherian $R$ module with $r\left(\operatorname{Ann}_{R}(M)\right)=\operatorname{Ann}_{R}(M)$, we show that $\Gamma(M)=E G(M)$ (Theorem 4.6), where $\Gamma(M)$ denotes the zero divisor graph of $M$.

\section{Properties of the essential graph for modules}

Let $R$ be a commutative ring and $M$ be an $R$-module. A submodule of $M$ is called essential if it has a non-trivial intersection with every non-trivial submodule of $M$.

Definition 2.1. Let $M$ be an $R$-module. The essential graph of $M$, denoted by $E G(M)$ is a simple undirected graph associated to $M$ with vertex set $Z(M) \backslash$ $\operatorname{Ann}_{R}(M)$, and a pair of distinct vertices $x$ and $y$ are adjacent if and only if $\operatorname{Ann}_{M}(x y)$ is an essential submodule of $M$.

Suppose that $x, y \in Z(M) \backslash \operatorname{Ann}_{R}(M)$. It is easy to see that $x$ and $y$ are adjacent in $E G(M)$ if and only if $\operatorname{Ann}_{M}(x)+\operatorname{Ann}_{M}(y)$ is an essential submodule of $M$.

Lemma 2.2. Let $M$ be an $R$-module. If $c \in r\left(\operatorname{Ann}_{R}(M)\right) \backslash \operatorname{Ann}_{R}(M)$, then $c$ is a universal vertex of $E G(M)$.

Proof. Let $c \in r\left(\operatorname{Ann}_{R}(M)\right) \backslash \operatorname{Ann}_{R}(M)$. Then $\operatorname{Ann}_{M}(c)$ is an essential submodule of $M$, by [5, Theorem 5(i)]. Hence, for each $a \in Z(M) \backslash \operatorname{Ann}_{R}(M), \operatorname{Ann}_{M}(a c)$ is an essential submodule of $M$. This means that $c$ is a universal vertex of $E G(M)$.

Lemma 2.3. Let $M$ be an $R$-module and let $c \in Z(M) \backslash \operatorname{Ann}_{R}(M)$ be a universal vertex of $E G(M)$. Then either $\operatorname{Ann}_{M}(c)$ is an essential submodule of $M$ or $R=$ $R_{1} \oplus R_{2}$ and $M=M_{1} \oplus M_{2}$, where $R_{1}$ and $R_{2}$ are subrings of $R, M_{1}$ and $M_{2}$ are $R$-submodules of $M$ and $(a, 0)$ is a universal vertex of $E G(M)$, for all $a \in Z_{R_{1}}\left(M_{1}\right)$.

Proof. Suppose that $c \in Z(M) \backslash \operatorname{Ann}_{R}(M)$ is a universal vertex of $E G(M)$. If $c^{2} M=0$, then the result follows by [5, Theorem 5(i)]. Suppose that $c^{2} M \neq 0$ and $c \neq c^{2}$. Thus $\operatorname{Ann}_{M}\left(c^{3}\right)$ is an essential submodule of $M$ so $\operatorname{Ann}_{M}(c)$ is an essential submodule of $M$.

Now, assume that $c^{2}=c$. Thus $R=c R \oplus(1-c) R$ and $M=c M \oplus(1-c) M$. Assume that $R_{1}=c R$ and $R_{2}=(1-c) R$. Then $R_{1}$ and $R_{2}$ are subrings of $R$. In addition, $M_{1}=c M$ and $M_{2}=(1-c) M$ are $R$-submodules of $M$. Moreover, if $r=\left(r_{1}, r_{2}\right)$ and $m=\left(m_{1}, m_{2}\right)$, then $r m=\left(r_{1} m_{1}, r_{2} m_{2}\right)$. It is easy to see that $c=(1,0)$. Then $(1,0)$ is a universal vertex of $E G(M)$. Assume that $0 \neq b \in$ $Z_{R_{2}}\left(M_{2}\right)$. Thus there exists $0 \neq m_{2} \in M_{2}$ such that $(1, b)\left(0, m_{2}\right)=(0,0)$ but 
$(1, b)\left(M_{1} \oplus M_{2}\right)=M_{1} \oplus b M_{2} \neq 0$. This means that $(1, b) \in Z(M) \backslash \operatorname{Ann}_{R}(M)$. Since $(1,0)$ is a universal vertex $\operatorname{Ann}_{M}((1,0)(1, b))=\operatorname{Ann}_{M}((1,0))=0 \oplus M_{2}$ is an essential submodule of $M$ that is impossible. Therefore, $Z_{R_{2}}\left(M_{2}\right)=0$. Moreover, if $a \in Z_{R_{1}}\left(M_{1}\right)$, then there exists $0 \neq m_{1} \in M_{1}$ such that $(a, 1)\left(m_{1}, 0\right)=(0,0)$ so $(a, 1) \in Z(M) \backslash \operatorname{Ann}_{R}(M)$. Thus $\operatorname{Ann}_{M}((1,0)(a, 1))=\operatorname{Ann}_{M}((a, 0))$ is an essential submodule of $M$, as required.

Remark 2.4. Let the situation be as Lemma 2.3. Since $\operatorname{Ann}_{M}((a, 0))$ is an essential submodule of $M$ so $\operatorname{Ann}_{M_{1}}(a)$ is an essential submodule of $M_{1}$. Moreover $R_{1}$ has characteristic 2 ; because, if $(1,0) \neq(-1,0)$, then $\operatorname{Ann}_{M}((1,0)(-1,0))=$ $\operatorname{Ann}_{M}((-1,0))=0 \oplus M_{2}$ is an essential submodule of $M$, that is impossible. Thus $1=-1 \in R_{1}$ and $R_{1}$ has characteristic 2 .

Theorem 2.5. Let $M$ be a Noetherian R-module with $r\left(\operatorname{Ann}_{R}(M)\right) \neq \operatorname{Ann}_{R}(M)$. Then $x, y \in Z(M) \backslash \operatorname{Ann}_{R}(M)$ are adjacent in $E G(M)$ if and only if $x y \in \mathfrak{p}$, for all $\mathfrak{p} \in \operatorname{MinAss}_{R}(M)$.

Proof. Suppose that $M$ is a Noetherian $R$-module and $\operatorname{MinAss}_{R}(M)=\left\{\mathfrak{p}_{1}, \cdots, \mathfrak{p}_{k}\right\}$. Thus there exists $m_{i} \in M$ such that $\mathfrak{p}_{i}=\operatorname{Ann}_{R}\left(m_{i}\right)$, for all $i=1, \cdots, k$. Assume that $x, y \in Z(M) \backslash \operatorname{Ann}_{R}(M)$ are adjacent in $E G(M)$ so $\operatorname{Ann}_{M}(x y)$ is an essential submodule of $M$. Hence, $\operatorname{Ann}_{M}(x y) \cap R m_{i} \neq 0$, for all $i=1, \cdots, k$. Therefore, $x y r_{i} m_{i}=0$ for some $r_{i} \in R$ with $0 \neq r_{i} m_{i}$ so $x y \in \mathfrak{p}_{i}$.

Conversely, suppose that $x y \in \mathfrak{p}$, for all $\mathfrak{p} \in \operatorname{MinAss}_{R}(M)$. We may assume that $x \in \cap_{j=1}^{t} \mathfrak{p}_{j}$ and $y \in \cap_{j=t+1}^{k} \mathfrak{p}_{j}$, for some $t$ with $1 \leq t \leq k$. So $x y \in r\left(\operatorname{Ann}_{R}(M)\right)=$ $\cap_{j=1}^{k} \mathfrak{p}_{j}$. Hence, $\operatorname{Ann}_{M}(x y)$ is an essential submodule of $M$ by Lemma 2.2. Therefore, $x$ and $y$ are adjacent in $E G(M)$, as needed.

Theorem 2.6. Let $M$ be an $R$-module such that $r\left(\operatorname{Ann}_{R}(M)\right) \neq \operatorname{Ann}_{R}(M)$. Then the following statements hold:

(i) $E G(M)$ is a connected graph with $\operatorname{diam}(E G(M)) \leq 2$.

(ii) If $M$ is Noetherian, then $\operatorname{gr}(E G(M)) \in\{3, \infty\}$.

Proof. (i) It is clear by Lemma 2.2 .

(ii) If $\left|r\left(\operatorname{Ann}_{R}(M)\right) \backslash \operatorname{Ann}_{R}(M)\right| \geq 2$, then either $E G(M)=K_{1,1}$ or $E G(M)$ has a cycle with length three, by Lemma 2.2 , so $\operatorname{gr}(E G(M))=3$. Now, assume that $r\left(\operatorname{Ann}_{R}(M)\right) \backslash \operatorname{Ann}_{R}(M)=\{c\}$. Two following cases may occur:

Case 1. Let $\operatorname{MinAss}_{R}(M)=\left\{\mathfrak{p}_{1}, \cdots, \mathfrak{p}_{k}\right\}(k \geq 2)$. Then for $x \in \mathfrak{p}_{i} \backslash \cup_{j=1, j \neq i}^{k} \mathfrak{p}_{j}$ and $y \in \cap_{j=1, j \neq i}^{k} \mathfrak{p}_{j} \backslash \mathfrak{p}_{i}$ we have $x \neq y, x, y \notin r\left(\operatorname{Ann}_{R}(M)\right)$ and $x y \in r\left(\operatorname{Ann}_{R}(M)\right)=$ $\cap_{j=1}^{k} \mathfrak{p}_{j}$. Hence, $x, y$ are adjacent in $E G(M)$ so $c-x-y-c$ is a cycle in $E G(M)$. 
Case 2. Let $\operatorname{MinAss}_{R}(M)=\{\mathfrak{p}\}$. If $x, y \in Z(M) \backslash r\left(\operatorname{Ann}_{R}(M)\right)$ are adjacent in $E G(M)$, then $\operatorname{Ann}_{M}(x y)$ is an essential submodule of $M$. So $\operatorname{Ann}_{M}(x y) \cap R m \neq 0$, where $\mathfrak{p}=\operatorname{Ann}_{R}(m)$ and $0 \neq m \in M$. Thus it is easy to see that either $x \in \mathfrak{p}$ or $y \in \mathfrak{p}$ which is a contradiction. Therefore, either $Z(M) \backslash r\left(\operatorname{Ann}_{R}(M)\right)=\{x\}$ and $E G(M)=K_{1,1}$ or $\left|Z(M) \backslash r\left(\operatorname{Ann}_{R}(M)\right)\right| \geq 2$ and $E G(M)=K_{1,\left|Z(M) \backslash r\left(\operatorname{Ann}_{R}(M)\right)\right|}$ that has no any cycle.

Corollary 2.7. Let $M$ be a Noetherian R-module with $r\left(\operatorname{Ann}_{R}(M)\right) \neq \operatorname{Ann}_{R}(M)$. If $\mathfrak{p}=r\left(\operatorname{Ann}_{R}(M)\right)$ is a prime ideal of $R$, then $E G(M)=K_{\left|\mathfrak{p} \backslash \operatorname{Ann}_{R}(M)\right|} \vee \bar{K}_{|Z(M) \backslash \mathfrak{p}|}$. In particular, $\operatorname{diam}(E G(M))=2$.

Proof. It is immediate by the proof of Theorem 2.6.

Corollary 2.8. Let $M$ be a Noetherian R-module with $r\left(\operatorname{Ann}_{R}(M)\right) \neq \operatorname{Ann}_{R}(M)$. Then $\operatorname{diam}(E G(M))=2$ if and only if there are $x, y \in Z(M) \backslash \operatorname{Ann}_{R}(M)$ and $\mathfrak{p} \in \operatorname{Ass}(M)$ such that $x y \notin \mathfrak{p}$.

Proof. It is immediate by Theorems 2.5 and 2.6 .

Lemma 2.9. Let $M$ be an $R$-module. Then $E G(M)$ is a complete graph if and only if one of the following statements holds:

(i) $\operatorname{Ann}_{M}(x)$ is an essential submodule of $M$, for all $x \in Z(M) \backslash \operatorname{Ann}_{R}(M)$.

(ii) $R=\mathbb{Z}_{2} \oplus \mathbb{Z}_{2}, M=\left(\oplus \mathbb{Z}_{2}\right) \oplus\left(\oplus \mathbb{Z}_{2}\right)$ and $Z(M)=\{(0,0),(1,0),(0,1)\}$.

Proof. Suppose that $E G(M)$ is complete and $x \in Z(M) \backslash \operatorname{Ann}_{R}(M)$. If either $x \in r\left(\operatorname{Ann}_{R}(M)\right)$ or $x \notin r\left(\operatorname{Ann}_{R}(M)\right)$ and $x \neq x^{2}$, then $\operatorname{Ann}_{M}(x)$ is an essential submodule of $M$, by Lemmas 2.2 and 2.3. Now, assume that $x \notin r\left(\operatorname{Ann}_{R}(M)\right)$ and $x=x^{2}$. Then, $R=R_{1} \oplus R_{2}$ and $M=M_{1} \oplus M_{2}$, where $R_{1}$ and $R_{2}$ are subrings of $R, M_{1}$ and $M_{2}$ are $R$-submodules of $M$ and $Z(M)=\left(Z_{R_{1}}\left(M_{1}\right) \oplus R_{2}\right) \cup\left(R_{1} \oplus 0\right)$, which follows from the proof of Lemma 2.3. If $0 \neq a \in Z_{R_{1}}\left(M_{1}\right)$, then there exists $0 \neq m_{1} \in M_{1}$ such that $(a, 1)\left(m_{1}, 0\right)=(0,0)$ so $(a, 1) \in Z(M) \backslash \operatorname{Ann}_{R}(M)$. Thus $\operatorname{Ann}_{M}((0,1)(a, 1))=\operatorname{Ann}_{M}((0,1))$ is an essential submodule of $M$, that is impossible. Hence, $Z(M)=\left(R_{1} \oplus 0\right) \cup\left(0 \oplus R_{2}\right)$. Let $(a, 0) \in\left(R_{1} \oplus 0\right) \backslash\{(0,0),(1,0)\}$. Then $(a, 0) \in Z(M) \backslash \operatorname{Ann}_{R}(M)$ and $\operatorname{Ann}_{M}((1,0)(a, 0))=\operatorname{Ann}_{M}((a, 0))=0 \oplus M_{2}$ is an essential submodule of $M$, that is impossible. So $R_{1}=\{0,1\}$ and $Z(M)=$ $\{(0,0),(1,0)\} \cup\left(0 \oplus R_{2}\right)$. By a similar argument one can show that $R_{2}=\{0,1\}$ and $Z(M)=\{(0,0),(1,0)\} \cup\{(0,0),(0,1)\}$ as desired. The converse is obvious.

Theorem 2.10. Let $M$ be a Noetherian R-module with $r\left(\operatorname{Ann}_{R}(M)\right) \neq \operatorname{Ann}_{R}(M)$. Then $E G(M)$ is a complete graph if and only if one of the following statements holds: 
(i) $Z(M)=r\left(\operatorname{Ann}_{R}(M)\right)$.

(ii) $E G(M)=K_{2}$.

Proof. Of course, (i) and (ii) imply that $E G(M)$ is a complete graph, see Lemma 2.2. Hence, it is enough to prove that if $E G(M)$ is complete, then either (i) or (ii) holds. Suppose that $E G(M) \neq K_{2}$ is a complete graph and $x \in Z(M) \backslash$ $\operatorname{Ann}_{R}(M)$. Then the increasing chain of submodules $\operatorname{Ann}_{M}(x) \subseteq \operatorname{Ann}_{M}\left(x^{2}\right) \subseteq$ $\ldots \subseteq \operatorname{Ann}_{M}\left(x^{n}\right) \subseteq \ldots$ does stabilize. Suppose that $n \in \mathbb{N}$ and $\operatorname{Ann}_{M}\left(x^{n}\right)=$ $\operatorname{Ann}_{M}\left(x^{n+i}\right)$, for all $i \geq 0$. If $m \in \operatorname{Ann}_{M}(x) \cap x^{n} M$, then $m=x^{n} m^{\prime}$, for some $m^{\prime} \in$ $M$. Hence, $x^{n+1} m^{\prime}=x m=0$ which implies that $m^{\prime} \in \operatorname{Ann}_{M}\left(x^{n+1}\right)=\operatorname{Ann}_{M}\left(x^{n}\right)$. So $m=0$ and then $x^{n} M=0$ since $\operatorname{Ann}_{M}(x)$ is an essential submodule of $M$. Therefore, $x \in r\left(\operatorname{Ann}_{R}(M)\right)$ and $Z(M)=r\left(\operatorname{Ann}_{R}(M)\right)$.

The following example has been presented to show that the property of being Noetherian is a necessary condition in Theorem 2.10.

Example 2.11. Let $p$ be a prime number and consider $\mathbb{Z}_{p^{\infty}}$ as a $\mathbb{Z}$-module. It is easy to see that $\operatorname{Ann}_{\mathbb{Z}_{p} \infty}\left(p^{i}\right)$ is an essential submodule of $\mathbb{Z}_{p^{\infty}}$, for all $i \geq 1$. Thus $E G\left(\mathbb{Z}_{p^{\infty}}\right)$ is a complete graph, but neither $Z_{\mathbb{Z}}\left(\mathbb{Z}_{p^{\infty}}\right)=r\left(\operatorname{Ann}\left(\mathbb{Z}_{p^{\infty}}\right)\right)$ nor $E G\left(\mathbb{Z}_{p^{\infty}}\right)=K_{2}$. Therefore, the Noetherian condition in Theorem 2.10 is necessary.

3. Results when $r\left(\operatorname{Ann}_{R}(M)\right)=\operatorname{Ann}_{R}(M)$

In this section, we investigate more results about the essential graph of $M$ whenever $r\left(\operatorname{Ann}_{R}(M)\right)=\operatorname{Ann}_{R}(M)$.

Lemma 3.1. Let $M$ be a Noetherian $R$-module such that $r\left(\operatorname{Ann}_{R}(M)\right)=\operatorname{Ann}_{R}(M)$ and let $0=\cap_{j=1}^{n} Q_{j}$ be a minimal primary decomposition of the zero submodule of $M$ with $r\left(\operatorname{Ann}_{R}\left(M / Q_{j}\right)\right)=\mathfrak{p}_{j}$, for each $j=1, \cdots, n$. Then the following statements hold:

(i) If $\mathfrak{p}_{i}$ is a minimal element of $\operatorname{Ass}_{R}(M)$, for some $i$ with $1 \leq i \leq n$, then there exists $a_{i} \in R$ such that $Q_{i}=\operatorname{Ann}_{M}\left(a_{i}\right)$.

(ii) If $\mathfrak{p}_{i}$ is a minimal element of $\operatorname{Ass}_{R}(M)$, for some $i$ with $1 \leq i \leq n$, then $Q_{i}$ is a prime submodule of $M$.

(iii) If $P=\operatorname{Ann}_{M}(a)$ is a prime submodule of $M$ and $\mathfrak{p}=\operatorname{Ann}_{R}\left(M / \operatorname{Ann}_{M}(a)\right)$, then $\mathfrak{p}$ is a minimal element of $\operatorname{Ass}_{R}(M)$.

(iv) If $P=\operatorname{Ann}_{M}(a)$ is a prime submodule of $M, \mathfrak{p}=\operatorname{Ann}_{R}\left(M / \operatorname{Ann}_{M}(a)\right)$ and $\mathfrak{p}=\mathfrak{p}_{i}$ for some $i$ with $1 \leq i \leq n$, then $P=Q_{i}$.

Proof. (i) Suppose that $0=\cap_{j=1}^{n} Q_{i}$ is a minimal primary decomposition of the zero submodule of $M$ with $r\left(\operatorname{Ann}_{R}\left(M / Q_{j}\right)\right)=\mathfrak{p}_{j}$, for each $j=1, \cdots, n$. Assume 
that $\mathfrak{p}_{i}=r\left(\operatorname{Ann}_{R}\left(M / Q_{i}\right)\right)$ is a minimal element of $\operatorname{Ass}_{R}(M)$ for some $i$ with $1 \leq i \leq$ $n$. Then $\cap_{j=1, j \neq i}^{n} \operatorname{Ann}_{R}\left(M / Q_{j}\right) \nsubseteq \mathfrak{p}_{i}$. Let $a_{i} \in \cap_{j=1, j \neq i}^{n} \operatorname{Ann}_{R}\left(M / Q_{j}\right) \backslash \mathfrak{p}_{i}$. We show that $\operatorname{Ann}_{M}\left(a_{i}\right)=Q_{i}$. Of course, we have $\operatorname{Ann}_{M}\left(a_{i}\right)=\left(0:_{M} a_{i}\right)=\left(\cap_{j=1}^{n} Q_{j}:_{M}\right.$ $\left.a_{i}\right)=\cap_{j=1}^{n}\left(Q_{j}:_{M} a_{i}\right)=\left(\begin{array}{ll}Q_{i}:_{M} & a_{i}\end{array}\right)$ and $Q_{i} \subseteq\left(Q_{i}:_{M} a_{i}\right)$. If $m \in\left(Q_{i}:_{M} a_{i}\right) \backslash Q_{i}$, then there exists $t \in \mathbb{N}$ such that $a_{i}^{t} M \subseteq Q_{i}$ so $a_{i} \in \mathfrak{p}_{i}$ which is a contradiction. Hence, $Q_{i}=\operatorname{Ann}_{M}\left(a_{i}\right)$.

(ii) From (i) it follows that $Q_{i}=\operatorname{Ann}_{M}\left(a_{i}\right)$, for some $a_{i} \in \cap_{j=1, j \neq i}^{n} \mathfrak{p}_{j} \backslash \mathfrak{p}_{i}$. We show that $Q_{i}$ is a prime submodule. Suppose that $b \in R, m \in M$ are such that $b m \in Q_{i}$ but $m \notin Q_{i}$. Thus there is $t \in \mathbb{N}$ such that $b^{t} M \subseteq Q_{i}$. So $\left(a_{i} b\right)^{t} M \subseteq Q_{i}$. On the other hand, $a_{i} b \in \cap_{j=1, j \neq i}^{n} \mathfrak{p}_{i}$ thus $\left(a_{i} b\right)^{t} M \subseteq \cap_{j=1, j \neq i}^{n} Q_{i}$. Hence, $\left(a_{i} b\right)^{t} M \subseteq$ $\cap_{j=1}^{n} Q_{j}=0$. Therefore, by the hypothesis $a_{i} b M=0$ so $b M \subseteq Q_{i}$ and $Q_{i}$ is prime.

(iii) Let $P=\operatorname{Ann}_{M}(a)$ be a prime submodule of $M$ and $\mathfrak{p}=\operatorname{Ann}_{R}\left(M / \operatorname{Ann}_{M}(a)\right)$. It is easy to see that $\mathfrak{p}=\operatorname{Ann}_{R}(a M)$. Let $m \in M$ and $a m \neq 0$. We show that $\mathfrak{p}=\operatorname{Ann}_{R}(a m)$. It is obvious that $\mathfrak{p} \subseteq \operatorname{Ann}_{R}(a m)$. Assume that $r \in R$ and ram $=0$. Thus $r m \in P=\operatorname{Ann}_{M}(a)$ and $m \notin P=\operatorname{Ann}_{M}(a)$ so $r a M=0$ and $r \in \operatorname{Ann}_{R}(a M)=\mathfrak{p}$. Hence, $\mathfrak{p}=\operatorname{Ann}_{R}(a m) \in \operatorname{Ass}_{R}(M)$. If $a \in \cap_{j=1}^{n} \mathfrak{p}_{j}$, then there is $t \in \mathbb{N}$ such that $a^{t} \in \cap_{j=1}^{n}\left(Q_{j}:_{R} M\right)$ so $a^{t} M \subseteq \cap_{j=1}^{n} Q_{j}=0$. Therefore, $a^{t} M=0$ and $a M=0$ which is a contradiction. Thus there are $1 \leq i \leq n$ and $\mathfrak{p}_{i} \in \operatorname{MinAss}_{R}(M)$ such that $a \notin \mathfrak{p}_{i}$. Assume that $r \in \mathfrak{p}$. Thus $r a M=0$ and so $r a M \subseteq \cap_{j=1}^{n} Q_{j}$. Hence, $r a \in\left(\cap_{j=1}^{n} Q_{j}:_{R} M\right) \subseteq \cap_{j=1}^{n}\left(Q_{j}:_{R} M\right) \subseteq \cap_{j=1}^{n} \mathfrak{p}_{j}$. Now, from $r a \in \mathfrak{p}_{i}$ and $a \notin \mathfrak{p}_{i}$ it follows that $\mathfrak{p} \subseteq \mathfrak{p}_{i}$ so $\mathfrak{p}=\mathfrak{p}_{i}$.

(iv) Suppose that $1 \leq i \leq n$ and $\mathfrak{p}=\mathfrak{p}_{i}$. We show that $P=Q_{i}$. Assume that $m \in Q_{i}$. Thus $a_{i} m=0 \in P$. If $m \notin P$, then $a_{i} \in \mathfrak{p}=\mathfrak{p}_{i}$, which is a contradiction so $Q_{i} \subseteq P$. Assume that $m \in P$ so $a m=0 \in Q_{i}$. If $m \notin Q_{i}$, then there is $s \in \mathbb{N}$ such that $a^{s} M \subseteq Q_{i}=\operatorname{Ann}_{M}\left(a_{i}\right)$. Hence, $a_{i} a^{s} M=0$ and so $a_{i} a^{s-1}(a M)=0$. Therefore, $a_{i} a^{s-1} \in \mathfrak{p}=\mathfrak{p}_{i}$ which implies that $a^{s-1} \in \mathfrak{p}=\mathfrak{p}_{i}$ since $a_{i} \notin \mathfrak{p}_{i}$. Hence, $a \in \mathfrak{p}$. This means that $a^{2} M=0$ and $a \in r\left(\operatorname{Ann}_{R}(M)\right)=\operatorname{Ann}_{R}(M)$ which is a contradiction. Therefore, $m \in Q_{i}$ and so $P \subseteq Q_{i}$.

Theorem 3.2. Let $M$ be a Noetherian R-module with $r\left(\operatorname{Ann}_{R}(M)\right)=\operatorname{Ann}_{R}(M)$. Then $E G(M)$ is a null graph if and only if $\left|\operatorname{MinAss}_{R}(M)\right|=1$.

Proof. $(\Leftarrow)$ Suppose that $a \in Z(M) \backslash \operatorname{Ann}_{R}(M)$ and $\operatorname{Ann}_{M}(a)$ is a prime submodule of $M$. Thus $\mathfrak{p}=\operatorname{Ann}_{R}(a M)$ is a minimal element of $\operatorname{Ass}_{R}(M)$, by Lemma 3.1(iii). In view of $\left[9\right.$, Lemma 3.2], $\operatorname{Ann}_{M}(a)$ is a unique maximal element of $X=\left\{\operatorname{Ann}_{M}(x): x \in Z(M) \backslash \operatorname{Ann}_{R}(M)\right\}$. So the zero submodule of $M$ has only one minimal primary component. Thus $r\left(\operatorname{Ann}_{R}(M)\right)=\operatorname{Ann}_{R}(M)=\mathfrak{p}$. Assume 
that $x$ and $y$ are adjacent vertices of $E G(M)$ so $\operatorname{Ann}_{M}(x y)$ is an essential submodule of $M$. If $\operatorname{Ann}_{M}(x y)$ is a proper submodule of $M$, then $\operatorname{Ann}_{M}(x y) \subseteq \operatorname{Ann}_{M}(a)$ implies that $\operatorname{Ann}_{M}(a)$ is an essential submodule of $M$, which contradicts [5, Theorem 5(iii)]. Hence, $\operatorname{Ann}_{M}(x y)=M$. So $x y \in \operatorname{Ann}_{R}(M)=\mathfrak{p}$ which implies that either $x \in \operatorname{Ann}_{R}(M)$ or $y \in \operatorname{Ann}_{R}(M)$ that is impossible. Therefore, $E G(M)$ is a null graph.

$(\Rightarrow)$ Since $M$ is a Noetherian $R$-module we have $\left|\operatorname{MinAss}_{R}(M)\right| \geq 1$. Moreover, $\left|\operatorname{MinAss}_{R}(M)\right|=|m-\operatorname{Ass}(M)| \leq 1$, by Lemma 3.1 and [5, Theorem 6(i)].

Theorem 3.3. [5, Theorem 7] Let $M$ be a Noetherian R-module and $\operatorname{Ann}_{R}(M)=$ $r\left(\operatorname{Ann}_{R}(M)\right)$. Then $E G(M)$ is a disconnected graph if and only if there exists $b \in Z(M) \backslash \operatorname{Ann}_{R}(M)$ such that $\operatorname{Ann}_{M}(b) \subseteq \bigcap_{P \in m-\operatorname{Ass}(M)} P$.

Corollary 3.4. Let $M$ be a Noetherian R-module with $r\left(\operatorname{Ann}_{R}(M)\right)=\operatorname{Ann}_{R}(M)$. Then $E G(M)$ is a connected graph if and only if for all $b \in Z(M) \backslash \operatorname{Ann}_{R}(M)$ there exists $P \in m-\operatorname{Ass}(M)$ such that $\operatorname{Ann}_{M}(b) \nsubseteq P$.

Corollary 3.5. Let $M$ be a Noetherian R-module. If $E G(M)$ is a connected graph, then $\operatorname{diam}(E G(M)) \leq 3$.

Proof. If $r\left(\operatorname{Ann}_{R}(M)\right) \neq \operatorname{Ann}_{R}(M)$, then the result follows by Theorem 2.6. Otherwise, it follows by Corollary 3.4, [5, Corollary 2] and [5, Remark 1(iii)], note that by Theorem $3.2,|m-\operatorname{Ass}(M)| \geq 2$.

Corollary 3.6. Let $M$ be a Noetherian R-module. If the connected graph $E G(M)$ has a cycle, then $\operatorname{gr}(E G(M)) \leq 4$.

Proof. If $r\left(\operatorname{Ann}_{R}(M)\right) \neq \operatorname{Ann}_{R}(M)$, then the result follows by Theorem 2.6. Now, assume that $r\left(\operatorname{Ann}_{R}(M)\right)=\operatorname{Ann}_{R}(M)$. For $\mid m$-Ass $(M) \mid \geq 3$ there is nothing to prove, see [5, Remark 1(iii)]. So we may assume that $|m-\operatorname{Ass}(M)| \leq 2$. On the other hand, $\mid m$-Ass $(M) \mid>1$ since $E G(M)$ is a connected graph, see Corollary 3.4. Hence, $|m-\operatorname{Ass}(M)|=2$. Now, by a similar argument to that of [11, Theorem 3.3] one can show that $\operatorname{gr}(E G(M)) \leq 4$.

Theorem 3.7. Let $M$ be a Noetherian R-module with $r\left(\operatorname{Ann}_{R}(M)\right)=\operatorname{Ann}_{R}(M)$ and assume that $E G(M)$ is not a star graph. Then $E G(M)$ is a complete bipartite graph if and only if $\left|\operatorname{Ass}_{R}(M)\right|=2$.

Proof. Let $I=\operatorname{Ann}_{R}(M)$. Note that

$$
r\left(\operatorname{Ann}_{R}(M)\right)=\operatorname{Ann}_{R}(M) \text { and } r\left(\operatorname{Ann}_{R}(M)\right) / I=r\left(\operatorname{Ann}_{R / I}(M)\right) .
$$


Thus $r\left(\operatorname{Ann}_{R / I}(M)\right)=0$. Moreover, for each $a \in R$, we have $a \in Z(M) \backslash \operatorname{Ann}_{R}(M)$ if and only if $a+I \in Z_{R / I}(M) \backslash\{0\}$ and $\mathfrak{p} \in \operatorname{Ass}_{R}(M)$ if and only if $\mathfrak{p} / I \in$ $\operatorname{Ass}_{R / I}(M)$. It is therefore enough for us to prove this result under the additional hypothesis that $r\left(\operatorname{Ann}_{R}(M)\right)=0$.

Let $E G(M)$ be a complete bipartite graph and $\left\{V_{1}, V_{2}\right\}$ be a partition of the vertex set of $E G(M)$. We prove that $V_{i} \cup\{0\}$ for $i=1,2$ is a prime ideal of $R$. Let $a, b \in \bar{V}_{1}=V_{1} \cup\{0\}$. If $a=0$ or $b=0$ or $a+b=0$, then $a+b \in \bar{V}_{1}$ and we are done. Suppose that $a, b \in V_{1}$. Thus there exist $x, y \in V_{2}$ such that $\operatorname{Ann}_{M}(a x)$ and $\operatorname{Ann}_{M}(b y)$ are essential submodules of $M$. If $\operatorname{Ann}_{M}(a x) \cap \operatorname{Ann}_{M}(b y) \subseteq \operatorname{Ann}_{M}(x y)$, then $\operatorname{Ann}_{M}(x y)$ is an essential submodules of $M$ which is a contradiction. Thus assume that $m \in \operatorname{Ann}_{M}(a x) \cap \operatorname{Ann}_{M}(b y) \backslash \operatorname{Ann}_{M}(x y)$. Hence, $(a+b) x y m=0$ and $x y m \neq 0$ this means $a+b \in Z(M)$. If $a+b \in V_{1}$ we are done; otherwise $a+b \in V_{2}$ and it follows that $\operatorname{Ann}_{M}(a(a+b))$ and $\operatorname{Ann}_{M}(b(a+b))$ are essential submodules of $M$. Then $\operatorname{Ann}_{M}\left((a+b)^{2}\right)$ and so $\operatorname{Ann}_{M}(a+b)$ is an essential submodule of $M$ which is a contradiction. So $a+b \in V_{1}$. Let $a, b \in R$ and $a b \in \bar{V}_{1}$. We show that either $a \in \bar{V}_{1}$ or $b \in \bar{V}_{1}$. If $a=0$ or $b=0$, then there is nothing to prove. If $a b=0$, then $\operatorname{Ann}_{M}(a b)$ is an essential submodule of $M$ contrary to the assumption. So assume that $0 \neq a, b, 0 \neq a b$ and $a, b \notin V_{1}$. Thus either $\operatorname{Ann}_{M}\left(a^{2} b\right)$ or $\operatorname{Ann}_{M}\left(a b^{2}\right)$ is an essential submodule of $M$ and so $\operatorname{Ann}_{M}\left((a b)^{2}\right)$ is an essential submodule of $M$ which implies that $\operatorname{Ann}_{M}(a b)$ is an essential submodule of $M$, this is a contradiction. Hence, either $a \in V_{1}$ or $b \in V_{1}$.

Conversely, assume that $\operatorname{Ass}_{R}(M)=\left\{\mathfrak{p}_{1}, \mathfrak{p}_{2}\right\}$. Thus $\mathfrak{p}_{1} \cap \mathfrak{p}_{2}=r\left(\operatorname{Ann}_{R}(M)\right)=$ 0 . Suppose that $a, b \in \mathfrak{p}_{1} \backslash\{0\}$ and $\operatorname{Ann}_{M}(a b)$ is an essential submodule of $M$. Moreover, suppose that $\mathfrak{p}_{2}=\operatorname{Ann}_{R}(m)$, for some $m \in M$. Thus $\operatorname{Ann}_{M}(a b) \cap R m \neq$ 0 . If $0 \neq r m \in \operatorname{Ann}_{M}(a b)$, then $a b r \in \mathfrak{p}_{2}$ which implies that $a b \in \mathfrak{p}_{2}$ and so either $a \in \mathfrak{p}_{2}$ or $b \in \mathfrak{p}_{2}$. Hence, either $a=0$ or $b=0$ which is a contradiction. Therefore, the elements of $\mathfrak{p}_{1} \backslash\{0\}$ are not adjacent with each other. By a similar argument, one can show that any two distinct elements of $\mathfrak{p}_{2} \backslash\{0\}$ are not adjacent. Let $a \in \mathfrak{p}_{1} \backslash\{0\}$ and $b \in \mathfrak{p}_{2} \backslash\{0\}$. Then $a b \in \mathfrak{p}_{1} \mathfrak{p}_{2} \subseteq \mathfrak{p}_{1} \cap \mathfrak{p}_{2}=0$ so $a b M=0$ which means that an element of $\mathfrak{p}_{1} \backslash\{0\}$ is adjacent to all elements of $\mathfrak{p}_{2} \backslash\{0\}$. Therefore, $E G(M)$ is a complete bipartite graph.

Corollary 3.8. Let $M$ be a Noetherian $R$-module with $r\left(\operatorname{Ann}_{R}(M)\right)=\operatorname{Ann}_{R}(M)$. Then $E G(M)$ is a star graph if and only if $R=\mathbb{Z}_{2} \oplus R^{\prime}$ and $M=\left(\oplus \mathbb{Z}_{2}\right) \oplus M^{\prime}$, where $R^{\prime}$ is a subring of $R$ and $M^{\prime}$ is an $R$-submodule of $M$ and $\operatorname{Ass}_{R}(M)=$ $\left\{\mathbb{Z}_{2} \oplus 0,0 \oplus R^{\prime}\right\}$. 
Proof. As in Theorem 3.7 we can assume that $r\left(\operatorname{Ann}_{R}(M)\right)=0$. Let $E G(M)$ be a star graph and let $\left\{V_{1}=\{c\}, V_{2}=\{x, y, z, \cdots\}\right\}$ be a partition of $V(E G(M))$. We prove that $V_{i} \cup\{0\}$ for $i=1,2$, is a prime ideal of $R$. By the hypotheses and the proof of Lemma 2.3 we have $c^{2}=c$ and also it follows that $R=R_{1} \oplus R_{2}$ and $M=M_{1} \oplus M_{2}$, where $R_{1}$ and $R_{2}$ are subrings of $R, M_{1}$ and $M_{2}$ are $R$-submodules of $M, c=(1,0)$ is the universal vertex of $E G(M), Z_{R_{1}}\left(M_{1}\right)=Z_{R_{2}}\left(M_{2}\right)=0$. Moreover, $R_{1}$ has characteristic 2 so $R_{1}=\mathbb{Z}_{2}$. Hence, $Z(M)=\mathbb{Z}_{2} \oplus 0 \cup 0 \oplus R_{2}$. Therefore, $V_{1} \cup\{0\}=\mathbb{Z}_{2} \oplus 0, V_{2} \cup\{0\}=0 \oplus R_{2}$ and $\operatorname{Ass}_{R}(M)=\left\{\mathbb{Z}_{2} \oplus 0,0 \oplus R_{2}\right\}$.

\section{Relations between the zero divisor graph and the essential graph}

In this section we will study the relations between the zero-divisor graph defined in [11] and the essential graph for modules.

Definition 4.1. [11, Definition 2.1] Let $M$ be an $R$-module. The zero-divisor graph of $M$, denoted by $\Gamma(M)$ is a simple undirected graph whose vertex set is $Z(M) \backslash \operatorname{Ann}_{R}(M)$ and two distinct vertices $x$ and $y$ are adjacent if and only if $x y M=0$.

To commence, we show that the zero-divisor graph is a subgraph of the essential graph.

Lemma 4.2. Let $M$ be an $R$-module. Then $\Gamma(M)$ is a subgraph of $E G(M)$.

Proof. Suppose that $x$ and $y$ are adjacent in $\Gamma(M)$. Then $x y M=0$ and $M=$ $\operatorname{Ann}_{M}(x y)$ is an essential submodule of $M$. Hence, $x$ and $y$ are adjacent in $E G(M)$.

Lemma 4.3. Let $M$ be an R-module and $x \in Z(M) \backslash r\left(\operatorname{Ann}_{R}(M)\right)$. If $\operatorname{Ann}_{M}(x)$ is a prime submodule of $M$, then $N_{\Gamma(M)}(x)=N_{E G(M)}(x)$.

Proof. Suppose that $x \in Z(M) \backslash r\left(\operatorname{Ann}_{R}(M)\right)$ and $\operatorname{Ann}_{M}(x)$ is a prime submodule of $M$. It is enough to show that $N_{E G(M)}(x) \subseteq N_{\Gamma(M)}(x)$. Assume that $y \in N_{E G(M)}(x)$. Thus $\operatorname{Ann}_{M}(x y)$ is an essential submodule of $M$. In view of [5, Theorem 5(iii)] $\operatorname{Ann}_{M}(x)$ is not an essential submodule of $M$. Hence, there exists a nonzero submodule $N$ of $M$ such that $\operatorname{Ann}_{M}(x) \cap N=0$. Therefore, for some $m \in M$ we have $x y m=0$ but $x m \neq 0$ so we get that $x y M=0 \operatorname{since}^{A_{n n}}(x)$ is a prime submodule of $M$. Therefore, $x$ and $y$ are adjacent in $\Gamma(M)$ and the proof is completed.

The following example shows that Lemma 4.3 does not hold necessarily for elements of $r\left(\operatorname{Ann}_{R}(M)\right)$. 
Example 4.4. Consider $M=\mathbb{Z} / 12 \mathbb{Z}$ as a $\mathbb{Z}$-module. For $6 \in r\left(\operatorname{Ann}_{R}(M)\right)$, $\operatorname{Ann}_{M}(6)=2 \mathbb{Z} / 12 \mathbb{Z}$ is a prime submodule of $M$ but $N_{\Gamma(M)}(6) \neq N_{E G(M)}(6)$.

Lemma 4.5. Let $M$ be a Noetherian R-module. Then 0 is a prime submodule of $M$ if and only if $E G(M)$ is a null graph. In particular, $E G(M)=\Gamma(M)$.

Proof. Suppose that 0 is a prime submodule of $M$. Then $\left|\operatorname{MinAss}_{R}(M)\right|=1$ and so the result follows by Theorem 3.2.

Theorem 4.6. Let $M$ be a Noetherian R-module with $r\left(\operatorname{Ann}_{R}(M)\right)=\operatorname{Ann}_{R}(M)$. Then $\Gamma(M)=E G(M)$.

Proof. It is obvious that $\Gamma(M)$ is a subgraph of $E G(M)$, by Lemma 4.2. Now, it is sufficient to show that each edge of $E G(M)$ is an edge of $\Gamma(M)$. Suppose that $x-y$ is an edge of $E G(M)$. Then $\operatorname{Ann}_{M}(x y)$ is an essential submodule of $M$. By the assumption the chain $\operatorname{Ann}_{M}(x y) \subseteq \operatorname{Ann}_{M}\left((x y)^{2}\right) \subseteq \ldots \subseteq \operatorname{Ann}_{M}\left((x y)^{n}\right) \subseteq$ ... of submodules does stabilize, thus there is $n \in \mathbb{N}$ such that $\operatorname{Ann}_{M}\left((x y)^{n}\right)=$ $\operatorname{Ann}_{M}\left((x y)^{n+i}\right)$, for all $i \geq 0$. Assume that $m \in \operatorname{Ann}_{M}(x y) \cap(x y)^{n} M$. Thus $m=(x y)^{n} m^{\prime}$ for some $m^{\prime} \in M$. Hence $(x y)^{n+1} m^{\prime}=x y m=0$, which implies that $m^{\prime} \in \operatorname{Ann}_{M}\left((x y)^{n+1}\right)=\operatorname{Ann}_{M}\left((x y)^{n}\right)$. Then $m=0$ and $(x y)^{n} M=0$ since $\operatorname{Ann}_{M}(x y)$ is an essential submodule of $M$. Therefore, $x y \in r\left(\operatorname{Ann}_{R}(M)\right)$ and so $x y M=0$.

The following examples have been presented to show that the properties of being Noetherian and $r\left(\operatorname{Ann}_{R}(M)\right)=\operatorname{Ann}_{R}(M)$ are necessary conditions in Theorem 4.6.

Example 4.7. $\quad$ (i) Example 2.11 shows that for the non-Noetherian $\mathbb{Z}$-module $\mathbb{Z}_{p^{\infty}}$ we have $r\left(\operatorname{Ann}_{\mathbb{Z}}\left(\mathbb{Z}_{p^{\infty}}\right)\right)=\operatorname{Ann}_{\mathbb{Z}}\left(\mathbb{Z}_{p^{\infty}}\right)$ but $E G\left(\mathbb{Z}_{p^{\infty}}\right) \neq \Gamma\left(\mathbb{Z}_{p^{\infty}}\right)$.

(ii) For the Noetherian $\mathbb{Z}$-module $\left.\mathbb{Z} / 12 \mathbb{Z}, \operatorname{Ann}_{\mathbb{Z}}(\mathbb{Z} / 12 \mathbb{Z})\right) \neq \operatorname{Ann}_{\mathbb{Z}}(\mathbb{Z} / 12 \mathbb{Z})$. The following figures (induced subgraphs of $\Gamma(\mathbb{Z} / 12 \mathbb{Z})$ and $E G(\mathbb{Z} / 12 \mathbb{Z})$ ) show that $E G(\mathbb{Z} / 12 \mathbb{Z}) \neq \Gamma(\mathbb{Z} / 12 \mathbb{Z})$.

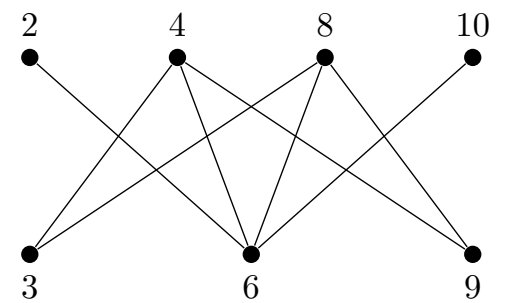

$\Gamma(M)$

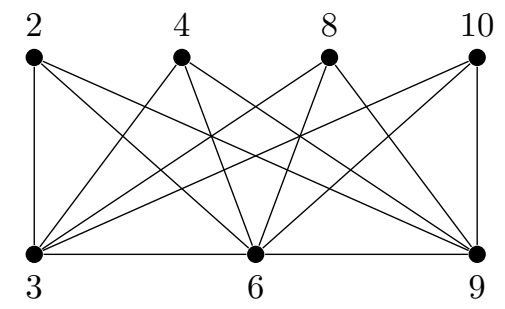

$E G(M)$ 
Acknowledgement. The authors would like to thank the referee for a careful reading of our paper and insightful comments which saved us from several errors.

\section{References}

[1] S. Akbari and A. Mohammadian, On the zero-divisor graph of a commutative ring, J. Algebra, 274 (2004), 847-855.

[2] D. F. Anderson and P. S. Livingston, The zero-divisor graph of a commutative ring, J. Algebra, 217(2) (1999), 434-447.

[3] D. F. Anderson and S. B. Mulay, On the diameter and girth of a zero-divisor graph, J. Pure Appl. Algebra, 210(2) (2007), 543-550.

[4] D. F. Anderson and D. Weber, The zero-divisor graph of a commutative ring without identity, Int. Electron. J. Algebra, 23 (2018), 176-202.

[5] S. Babaei, Sh. Payrovi and E. Sengelen Sevim, On the annihilator submodules and the annihilator essential graph, Acta Math. Vietnam., 44 (2019), 905-914.

[6] I. Beck, Coloring of commutative rings, J. Algebra, 116(1) (1988), 208-226.

[7] M. Behboodi, Zero divisor graphs for modules over commutative rings, J. Commut. Algebra, 4(2) (2012), 175-197.

[8] S. C. Lee and R. Varmazyar, Zero-divisor graphs of multiplication modules, Honam Math. J., 34(4) (2012), 571-584.

[9] C. P. Lu, Unions of prime submodules, Houston J. Math., 23(2) (1997), 203213.

[10] M. J. Nikmehr, R. Nikandish and M. Bakhtyiari, On the essential graph of a commutative ring, J. Algebra Appl., 16(7) (2017), 1750132 (14 pp).

[11] K. Nozari and Sh. Payrovi, A generalization of zero-divisor graph for modules, Publ. Inst. Math. (Beograd) (N.S.), 106(120) (2019), 39-46.

[12] S. Safaeeyan, M. Baziar and E. Momtahan, A generalization of the zero-divisor graph for modules, J. Korean Math. Soc., 51(1) (2014), 87-98.

[13] R. Y. Sharp, Steps in Commutative Algebra, Second edition, Cambridge University Press, Cambridge, 2000.

F. Soheilnia, Sh. Payrovi (Corresponding Author), and A. Behtoei

Department of Mathematics

Imam Khomeini International University

P. O. Box: 3414916818, Qazvin, Iran

e-mails: f.soheilnia@edu.ikiu.ac.ir (F. Soheilnia)

shpayrovi@sci.ikiu.ac.ir (Sh. Payrovi)

a.behtoei@sci.ikiu.ac.ir (A. Behtoei) 